\title{
Treatment of Polycystic Ovarian Syndrome using Electroacupuncture: A Case Report
}

\author{
Fariba Farajzadeh ${ }^{1}$, Fariba Ghaderi ${ }^{*}$, Parvin Bastani ${ }^{3}$
}

\begin{abstract}
Objectives: Polycystic ovarian syndrome (PCOS) with symptoms of hyperandrogenism is one of the most common endocrine disorders of women at reproductive age.

Case Presentation: The patient was a 19-year-old woman complaining of menstrual irregularities and was diagnosed with PCOS 5 years ago. The medications prescribed for 4 years had no significant therapeutic effect. The patient had menstrual irregularity and amenorrhea in the last 3 months. Ultrasound examination revealed bilateral polycystic ovaries. She underwent electroacupuncture (EA) for 14 sessions during 4 months in $\mathrm{LI}_{4}, \mathrm{SP}_{6}, \mathrm{Ren}_{19}, \mathrm{ST}_{36}$, and $\mathrm{CV}_{6}$ acupuncture points. According to ultrasound examination the polycystic view of ovaries disappeared in the 16th weeks of follow-up; sign of ovulation was observed in the ovaries and endometrial thickness increased. The ratio of luteinizing hormone (LH) to follicle stimulating hormone (FSH) reduced, and her most important complaint, i.e., menstrual irregularities, was almost completely eliminated, and the regular menstruation still continued up to six months after the last session of treatment.

Conclusion: Use of EA improved patient symptoms through reducing sympathetic tone in the arteries of uterine and ovaries as well as reflexive increasing of circulation through low-frequency contractions in the muscles.

Keywords: Polycystic ovarian syndrome (PCOS), Acupuncture, Electroacupuncture
\end{abstract}

\section{Introduction}

Polycystic ovarian syndrome (PCOS) is one of the most common ovarian disorders in women and affects the function of female reproductive system (1). Its incidence was reported as $15.2 \%$ in Iran based on the Rotterdam criteria (2).

Given the high prevalence of disorders such as hyperandrogenism, hirsutism, impairment of ovulation and fertility arising from this condition, any treatment that can help to treat these symptoms is important (1).

Since techniques such as acupuncture or stimulating the acupuncture points are new for Iranian population, these treatment methods are barely accepted by physicians and patients especially in unusual cases such as PCOS, unlike societies such as China that have a long history in this area.

Therefore, this report may help changing the view of specialists towards the non-invasive and non-pharmacological treatment of the disease.

PCOS is a functional disorder in the growth and development of ovarian follicles leading to chronic anovulation (1).

Clinical presentation of this syndrome includes hyperandrogenic symptoms like: hirsutism, acne, and androgenic alopecia as well as ovarian symptoms such as menstrual irregularities and amenorrhea. Increased serum levels of luteinizing hormone $(\mathrm{LH})$ to follicle stimulating hormone (FSH) are a paraclinical symptom of PCOS (1). According to Rotterdam criteria, the following three main diagnostic criteria are: 1) low ovulation or anovulation, 2) clinical and biochemical symptoms of hyperandrogenism, and 3) multiple primary follicles in the ovaries determined by ultrasound. Two of three diagnostic criteria are enough for diagnosis (1).

This common gynecologic problem is treated by invasive (including surgery), and non-invasive methods. Lifestyle changes, medications, exercise therapy, and acupuncture are such conservative treatments.

For regulating the menstrual cycle, estrogen and progesterone based drugs are used, while if inducing pregnancy is the aim of treatment, metformin, clomifen citrate, letrozole, and injective gonadotropins are used.

Electroacupuncture (EA) which uses electrical stimulation in the acupuncture points is one of the methods of complementary medicine for these patients and has no side effect. This method improves ovulation in these patients through affecting secretion of beta-endorphins in the central nervous system and inhibition of sympathetic tone. Also EA increases uterine blood flow resulting in reduction of uterine vasoconstriction by sympathetic nervous system (3-5).

Repeated EA sessions decrease testosterone and the ratio of $\mathrm{LH}$ to $\mathrm{FSH}$, and ultimately improve menstrual 
regularity in women with PCOS complaining from amenorrhea or irregular menstruation, with no side effects $(5,6)$. Applying acupuncture or acupressure can stimulate acupuncture points but EA increases the impact of needles in the acupuncture points, so we used EA instead of acupuncture for this patient. The aim of this study was to evaluate the effects of EA for PCOS treatment in a patient with failed previous drug therapy.

\section{Case Presentation}

The studied patient was a single 19-year-old woman with complaints of impaired menstrual cycles for 5 years and amenorrhea in the past three months. She was diagnosed with PCOS and received different combinations of estrogen and progesterone.

The patient weighed $65 \mathrm{~kg}$, her height and waist-hip ratio (WHR) were $165 \mathrm{~cm}$ and 74 to 59 , respectively, and she was not overweight.

According to the results of ultrasound, the right ovary had a small cyst $(21 \times 31 \mathrm{~mm})$ and the left ovary was polycystic, and the endometrial thickness was $4.6 \mathrm{~mm}$.

LH to FSH ratio was 10.7 to 8.15 and slightly increased based on the existing criteria. The validated Persian version of quality of life questionnaire (7) in patients with PCOS was used in this study and a score of 130 out of 210 was obtained. The maximal score in this questionnaire shows maximal involvement due to PCOS signs and symptoms. According to the questionnaire, the patient did not have the symptoms of hirsutism, while she suffered from hair loss and acne, irregular menstrual cycles, depression, and anxiety.

Due to dissatisfaction of the patient with medication in recent years, she received a full explanation of EA and agreed to use this method. Informed consent was obtained from the patient and specialists were consulted to rule out cardiovascular disease, tumors, endocrine causes, diabetes, Cushing's syndrome, congenital adrenal hyperplasia, and hyperprolactinemia and to confirm the treatment method of EA.

\section{Management and Outcome}

Treatment with EA was performed using an E-160 Device (ITO, Japan). Electrical stimulation with duration of $300 \mathrm{~ms}$ was applied bilaterally in points $\mathrm{LI}_{4}, \mathrm{SP}_{6}, \mathrm{Ren}_{19}$, $\mathrm{ST}_{36}$, and $\mathrm{CV}_{6}$ (Figure 1) with a frequency of $2 \mathrm{~Hz}$ for 30 minutes.

According to available studies $(5,6,8)$, the therapy was performed twice a week in the first 2 weeks, once a week in the next 6 weeks, and once per two weeks in the 8 next weeks. Duration and frequency were not changed over 14 sessions in 4 months.

According to recommendations of the available studies, the patient was reassessed after 16 weeks.

The results of ultrasound examination performed during the follicular phase of the menstrual cycle showed the right ovarian cyst had completely disappeared and the left ovary did not have a polycystic view. The endometrial
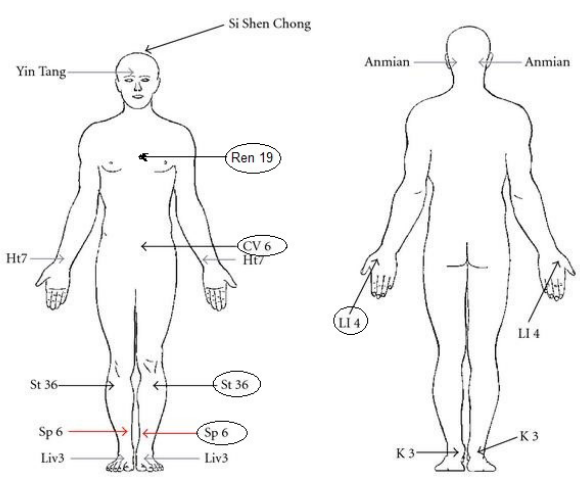

Figure 1. Acupressure point locations: Conception Vessel 6 (CV6), Large Intestine 4 (LI4), Stomach 36 (St36), Spleen 6 (Sp6), Ren 19.

thickness increased to $9.9 \mathrm{~mm}$. Unlike the previous ultrasound, follicles in the ovaries were reported at a suitable level.

The quality of life questionnaire score was reduced from 130 to 74 , which was recovered about $27 \%$ in comparison with baseline. Scores of facial acne, regularity of menstrual cycles, depression, and anxiety were significantly improved.

Follow up evaluation six months later showed a stable improvement in menstrual cycles as the most important complaint and concern of the patient. The quality of life questionnaire score was still 75 like at the end of the treatment.

\section{Discussion}

The studied patient with a history of PCOS in the past 5 years was received 14 sessions of EA during 16 weeks, and the symptoms in ultrasound examination including reduced number of primary follicles and increased endometrial thickness as well as clinical signs such as acne, menstrual irregularity, and PCOS-induced depression significantly improved.

Studies in this field pointed out effective reduction of PCOS-induced hyperandrogenism due to low-frequency EA (9-11).

The patient did not improve during 4 years of treatment with oral contraceptives. On the other hand, these hormones disrupted her metabolic function (8).

The use of low-frequency EA can stably improve menstrual irregularity and reduce acne; according to previous studies (9-11) and 6 months following the studied patient showed the menstrual cycle was still regular.

Applying low-frequency EA with the parameters used in this study led to improvement of PCOS inducing depression and anxiety; this indicator was also improved in the study of Billhult A, Stener-Victorin. They attributed this improvement in 8 studied women to the nature of acupuncture which initially leads to pain and discomfort, increased tension, and then relaxation and trust in the therapist (9). It should be noted that the majority of these patients, like the case in the present study, have examined 
different medical methods without any improvement. Therefore, most of them were despaired, but after reliance on this method and observing improvement of clinical signs such as acne and hirsutism as well as irregular menstrual cycles, their confidence and satisfaction increased. After observing the effects of treatment, these patients also got interested to continue the treatment completely (9).

Ultrasound showed ovulation after EA in the patient; this was also seen in the study of Stener-Victorin et al. Increased muscle sympathetic activity (MSNA) in patients with PCOS has been shown in various studies. In the study of Stener-Victorin et al on 20 women with PCOS, a significant decrease in MSNA was observed in two out of three groups, i.e., in the EA and exercise groups compared to the control group (11).

It was shown in their study that women with PCOS with lower body mass index (BMI) and WHR respond better to EA. As in the present study the patient with a BMI of 23.89 had a good response to the treatment.

According to the study of $\mathrm{Yu}$ et al (10), the mechanism of increased ovulation by EA is unknown. However, based on a hypothesis, the release of gonadotropin-releasing hormone $(\mathrm{GnRH})$ decreases in PCOS due to reduced inhibition of beta-endorphine or increased sympathetic activity in the ovaries.

EA is effective on PCOS both psychologically and physiologically. Low-frequency EA stimulates muscle mechanoreceptors and surrounding tissues. This process is activated by EA and muscle contractions. EA and muscle exercise can release beta-endorphine by two mechanisms; first hypothalamus and nervous system inhibit vasomotor and sympathetic nervous system in brain stem nuclei and midbrain. The second mechanism is concurrent release of beta-endorphin and adrenocorticotropic hormone $(\mathrm{ACTH})$ in the blood. Both systems reduce sympathetic tone through release of beta-endorphine and affect PCOS (8-11).

Another important finding of this study was the significant increase in endometrial thickness in the studied patient, which was evident in the ultrasound results. This finding in patients with PCOS who suffer from infertility and infertile women due to reduced endometrial thickness is so important, because it can highly affect their successful fertility or possibility of successful intrauterine insemination (IUI) and in vitro fertilization (IVF). This finding has not been reported in the literature about PCOS, but it is predictable, given the decreased MSNA of uterus. This finding has been reported in the studies of infertility and increased possibility of IVF $(12,13)$.

As discussed EA can be used in treatment of PCOS especially in patients with previous failed drug treatment and enough motivation to continue the EA treatment.

\section{Ethical Issues}

The study was approved by the ethical committee of Tabriz University of Medical Science. Additionally, written informed consent was obtained.

\section{Acknowledgments}

The authors would like to give his gratitude to the studied patient (A.F.), the Department of Physical Therapy, Rehabilitation Faculty, and the Department of Obstetrics and Gynecology, Medical School, Tabriz University of Medical Sciences.

\section{Conflict of interests}

None declared.

\section{Financial Support}

None.

\section{References}

1. Fritz MA, Speroff L. Clinical Gynecologic Endocrinology and Infertility. 8th ed. Philadelphia: Lippincott Williams \& Wilkins; 2011.

2. Mehrabian E, Khani B, Kelishadi R. The prevalence of polycystic ovary syndrome in Iranian women based on different diagnostic criteria. Polish J Endocrinol. 2011; 62(3):238-242.

3. Stener-Victorin E, Waldenstrom U, Andersson SA, Wikland M. Reduction of blood flow impedance in the uterine arteries of infertile women with electroacupuncture. Hum Reprod. 1996;11(6):1314-1317.

4. Stener-Victorin E, Jedel E, Janson PO, Sverrisdottir YB. Low-frequency electroacupuncture and physical exercise decrease high muscle sympathetic nerve activity in polycystic ovary syndrome. Am J Physiol Regul Integr Comp Physiol. 2009;297:R387-R395.

5. Stener-Victorin E, Waldenstrom U, Tagnfors U, Lundeberg T, Lindstedi G, Janson PO. Effects of electro-aclupuncture on anovulation in women with polycystic ovary syndrome. Acta obstet Gynecol Scand. 2000;79:180-188.

6. Chen BY, Yu J. Relationship between blood radio immune-reactive beta-endorphin and hand skin temperature during the electro-acupuncture induction of ovulation. Acupunct Electrother Res. 1991;16:1-5.

7. Bazarganipour F, Ziaei S, Montazeri A, Foroozanfard F, Faghihzadeh S. Iranian version of modified polycystic ovary syndrome health-related quality of life questionnaire: discriminant and convergent validity. Iran J Reprod Med. 2013;9:753-760.

8. Jedel E, Labrie F, Oden A, et al. Impact of electroacupuncture and physical exercise on hyper androgenism and oligo/amenorrhea in women with polycystic ovary syndrome: a randomized controlled trial. Am J Physiol Endocrinol Metab. 2011;300(1):E37-E45. doi:10.1152/ ajpendo.00495.2010.

9. Billhult A, Stener-Victorin E. Acupuncture with manual and low frequency electrical stimulation as experienced by women with polycystic ovary syndrome: a qualitative study. BMC Complement Altern Med. 2012;12:32. doi:10.1186/1472-6882-12-32.

10. Yu L, Liao Y, Wu H, et al. Effects of electroacupuncture and Chinese kidney-nourishing medicine on polycystic ovary syndrome in obese patients. J Tradit Chin Med. 
2013;33(3):287-293.

11. Stener-Victorin E, Baghaei F, Holm G, et al. Effects of acupuncture and exercise on insulin sensitivity, adipose tissue characteristic, and markers of coagulation and fibrinolysis in women with polycystic ovary syndrome: Secondary analyses of a randomized controlled trial. Fertil Steril. 2012;97(2):501-8. doi:10.1016/j. fertnstert.2011.11.010.
12. Ho M, Huang LC, Chang YY, et al. Electroacupuncture reduces uterine artery blood flow impedance in infertile women. Taiwan J Obstet Gynecol. 2009;48(2):148-51. doi:10.1016/S1028-4559(09)60276-X.

13. Anderson BJ, Haimovici F, Ginsburg ES, Schust DJ, Wayne PM. In vitro fertilization and acupuncture: clinical efficacy and mechanistic basis. Altern Ther Health Med. 2007;13(3):38-48.

Copyright (c) 2017 The Author(s); This is an open-access article distributed under the terms of the Creative Commons Attribution License (http://creativecommons.org/licenses/by/4.0), which permits unrestricted use, distribution, and reproduction in any medium, provided the original work is properly cited. 